\title{
Towards the theory of boundary value problems on non-smooth manifolds
}

Cite as: AIP Conference Proceedings 2325, 020002 (2021); https://doi.org/10.1063/5.0040277

Published Online: 09 February 2021

\section{Vladimir Vasilyev}
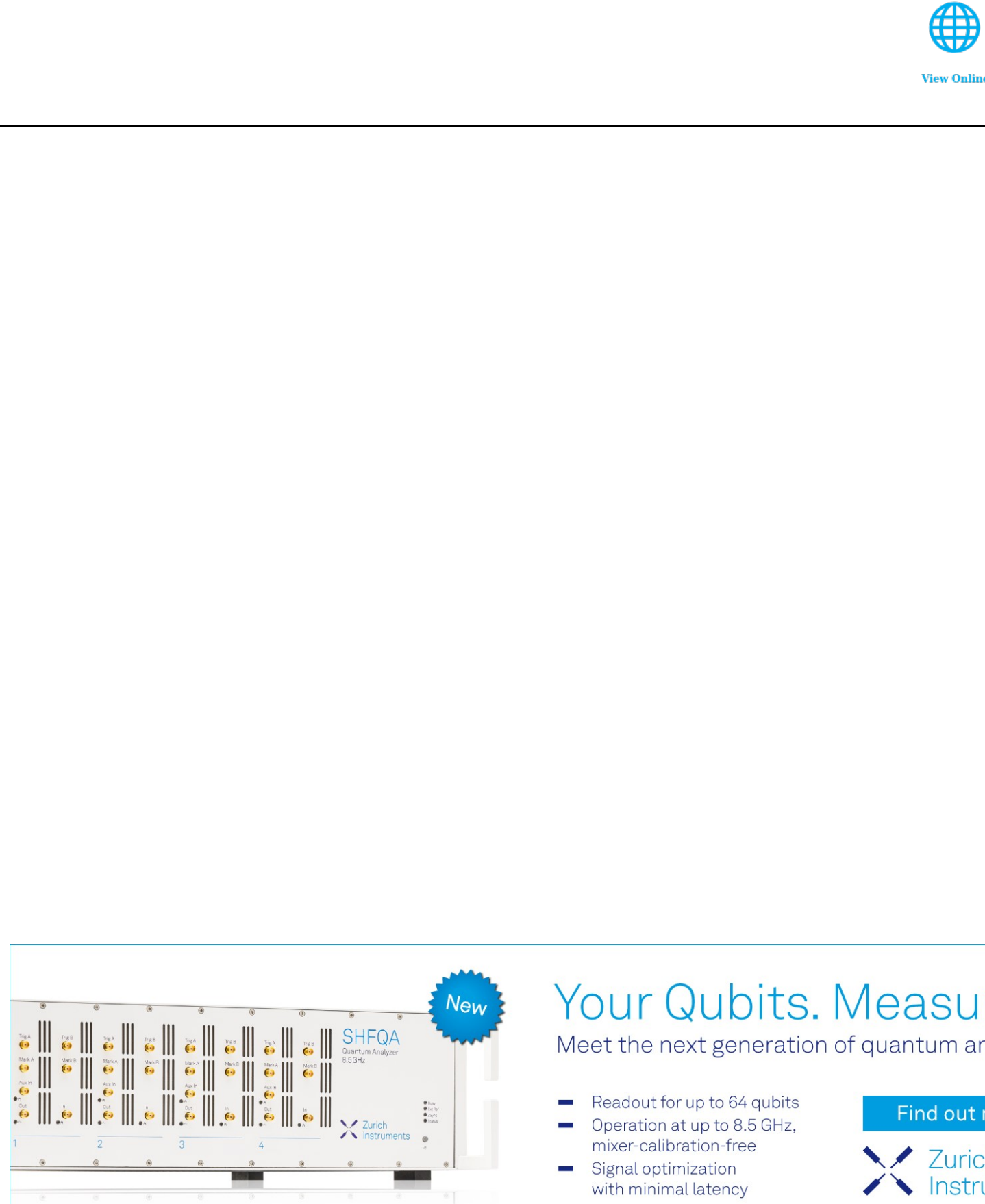

\section{Your Qubits. Measured.}

Meet the next generation of quantum analyzers

- Readout for up to 64 qubits

- Operation at up to $8.5 \mathrm{GHz}$. mixer-calibration-free

- Signal optimization with minimal latency 


\title{
Towards the Theory of Boundary Value Problems on Non-Smooth Manifolds
}

\author{
Vladimir Vasilyev ${ }^{\text {a) }}$ \\ Chair of Applied Mathematics and Computer Modeling, Belgorod State National Research University, Pobedy street 85, Belgorod \\ 308015, Russia

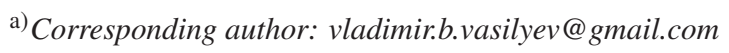

\begin{abstract}
We suggest some constructions to develop the theory of boundary value problems on manifolds with a non-smooth boundaries. We discuss basic principles for such a theory and describe main results that we have obtained to this time. Further, we show how these results are related to the theory of boundary value problems on non-smooth manifolds.
\end{abstract}

\section{INTRODUCTION}

In this paper we would like to present basic ideas and results which can be useful for constructing the theory of boundary value problems for elliptic pseudo-differential equations on manifolds with a non-smooth boundary.

There are a lot of books $[1,2,3,4,5,6]$ in which similar problems are discussed but they use distinct methods and consider certain "singular manifolds" of other types.

Some papers are very closed to this text $[7,8,9]$ but these are related to algebraical aspects of such a theory.

The paper [10] is classified as asymptotical aspect of such a theory, some author's studies and results can be found in $[11,12]$.

Author's results were presented in the book [13]. Generally these results concerned to the two-dimensional situation. Here we present our main ideas and results which are under consideration last years $[11,12,14,15,16,17,18]$ and these latter considerations are related to multi-dimensional case especially. Here we continue these studies and give certain concrete constructions for special boundary value problems.

\section{BASIC PRINCIPLES}

We use a few basic principles on which the theory holds. We will describe briefly all these postulates because these are very simple and give the key for understanding the theory. As a model we will take the following operator

$$
u(x) \longmapsto \int_{D} A(x, x-y) u(y) d y, \quad x \in D,
$$

where $A(x, y)$ is "kernel" of the operator, i.e. $A(x, y)=F_{\xi \rightarrow y}^{-1} \tilde{A}(x, \xi), F$ is the Fourier transform,

$$
\tilde{u}(\xi)=(F u)(\xi)=\int_{\mathbb{R}^{m}} e^{i x \cdot \xi} u(x) d x,
$$

$D \subset \mathbb{R}^{m}$ is a bounded domain (for simplicity) with a boundary $\partial D$. Such operator (1) is called a pseudo-differential operator in $D[19]$ with the symbol $\tilde{A}(x, \xi)$.

We will suppose here that the symbol $A(x, \xi)$ satisfies some smoothness properties (for example, $A(x, \xi)$ is continuously differentiable on $\bar{D} \times \mathbb{R}^{m}$ ) and the condition

$$
c_{1}(1+|\xi|)^{\alpha} \leq|A(x, \xi)| \leq c_{1}(1+|\xi|)^{\alpha}, \quad \alpha \in \mathbb{R} .
$$

Let us note the latter condition guarantees the boundedness of the operator (1) in Sobolev-Slobodetskii spaces $H^{s}(D) \rightarrow H^{s-\alpha}(D)[19]$.

The main problem of the theory is describing Fredholm properties of the operator (1).

Definition 1 Operator (1) is called an elliptic operator if

$$
\text { ess } \inf _{x \in \bar{D}, \xi \in \mathbb{R}^{m}}|A(x, \xi)|>0 .
$$

International Conference on Analysis and Applied Mathematics (ICAAM 2020) AIP Conf. Proc. 2325, 020002-1-020002-10; https://doi.org/10.1063/5.0040277 Published by AIP Publishing. 978-0-7354-4069-2/\$30.00 
Such symbol is called elliptic symbol.

It is convenient considering operators (1) in Sobolev-Slobodetskii spaces $H^{s}(D)$ [19]. We will remind the definition [19]. By definition, this space consists of functions (distributions) $u$ from $H^{s}\left(\mathbb{R}^{m}\right)$ which supports belong to $\bar{D}$. A norm in the space $H^{s}(D)$ is induced by the $H^{s}\left(\mathbb{R}^{m}\right)$-norm

$$
\|u\|_{s}=\left(\int_{\mathbb{R}^{m}}|\tilde{u}(\xi)|^{2}(1+|\xi|)^{2 s} d \xi\right)^{1 / 2},
$$

where the sign $\sim$ over $u$ denotes its Fourier transform

$$
\tilde{u}(\xi)=\int_{\mathbb{R}^{m}} u\left(x \left(e^{i x \cdot \xi} d x\right.\right.
$$

Everywhere below we consider only elliptic symbols, and $\widetilde{H}(D)$ denotes the Fourier image of the space $H(D)$.

\section{Local principle}

This principle was introduced by I.B. Simonenko [20] more than a half-century ago. It asserts in a simplest form that an operator of a special type operator of a local type) has Fredholm property iff all certain other operators (its local representatives) have the same property. It seems there is no news but the essential point is that local representative of an operator can have more simple structure than the starting general operator. As we will see below it is real fact.

\section{Complicated Operator at a Boundary Point}

This concept is related to the first principle. We will explain it for the case when the boundary $\partial D$ is a smooth surface. This case includes two types of local representatives. If the point $x_{0} \in D$ is an inner point then a neighborhood of the point is diffeomorphic to $\mathbb{R}^{m}$ and the local representative of the the operator (1) looks as follows

$$
u(x) \longmapsto \int_{\mathbb{R}^{m}} A\left(x_{0}, x-y\right) u(y) d y, \quad x \in \mathbb{R}^{m} .
$$

To obtain Fredholm property (= invertibility property) for such operator(2) in "local" Sobolev-Slobodetskii space $H^{s}\left(\mathbb{R}^{m}\right)$ it is necessary and sufficient to require the ellipticity condition $[19,20]$.

If the point $x_{0} \in \partial D$ is a boundary point then a neighborhood of the the point is diffeomorphic to half-space $\mathbf{R}_{+}^{m}=\left\{x \in \mathbf{R}^{m}: x=\left(x_{1}, \ldots, x_{m}\right), x_{m}>0\right\}$ (rectification of a boundary), and the local representative of the the operator (ref1) looks as follows

$$
u(x) \longmapsto \int_{\mathbb{R}_{+}^{m}} A\left(x_{0}, x-y\right) u(y) d y, \quad x \in \mathbb{R}_{+}^{m}
$$

The latter operator (3) acts in "local" Sobolev-Slobodetskii spaces $H^{s}\left(\mathbb{R}_{+}^{m}\right)$. Such operators (3) are called usually Wiener-Hopf operators. The ellipticity condition for their invertibility is not enough. Principal role takes factorization technique based on classical Riemann boundary value problem [19]. The dimension of a kernel (co-kernel) of the operator (3) is defined by so called index of factorization. We denote it by ${ }_{m-1}\left(x_{0}\right)$, generally it depends on the point $x_{0} \in \partial D$.

\section{Conjugation Problem and Factorizability Principle at a Boundary Point}

This idea was applied by the author earlier [13] and it related to a new type of local representatives of the operator (1). They appear when the boundary $\partial D$ includes some singular points in which smoothness property does not hold. 
For example, if $x_{0} \in \partial D$ such that its neighborhood is diffeomorphic to a cone $C \subset \mathbb{R}^{m}$ and a neighborhood of $\partial D$ transforms to a neighborhood of $\partial C$ then local representative of the operator (1) is the following

$$
u(x) \longmapsto \int_{C} A\left(x_{0}, x-y\right) u(y) d y, \quad x \in C,
$$

and we need to study invertibility properties of the operator (4) in "local" Sobolev-Slobodetskii space $H^{s}(C)$. Therefore, if you want to do it or not you must invent how to find the inverse operator for (4).

\section{Multidimensional Riemann boundary value problem and the wave factorization}

For this purpose the author has studied a certain multidimensional variant of Riemann boundary value problem [13]. It appears if we try to apply the Fourier transform to study the operator (4). As a result we obtain some multidimensional singular integral equation with a special kernel; this equation is a generalization of one-dimensional singular integral equation with the Cauchy kernel.

Bochner kernel Let $C$ be a convex cone non-including a whole straight line. This kernel is defined by the formula

$$
B(z)=\int_{C} e^{i y \cdot z} d y, \quad z=x+i \tau, \quad \tau \in \stackrel{*}{C}
$$

where $\stackrel{*}{C}=\left\{x \in \mathbf{R}^{m}: x \cdot y>0, y \in C\right\}$. The Bochner kernel is holomorphic function in radial tube domain $T\left({ }^{*}\right)=$ $\mathbf{R}^{m}+i \stackrel{*}{C} \subset \mathbf{C}^{m}[21,22,23]$. Only one cone exists in the real line, and for this case the Bochner kernel transforms into Cauchy kernel.

Conjugation problem Up to now there is no any way to solve such singular integral equations excluding the Wiener-Hopf method (= method of Riemann boundary value problem). This method is based on a special representation for coefficient of conjugation problem (or in other words for the symbol of a "local operator"). The author has used this idea for the multidimensional Riemann boundary value problem and has introduced the concept of wave factorization for an elliptic symbol.

If we consider the cone $\mathbb{R}^{k} \times C^{m-k}$ where $C^{m-k}$ is a convex cone in $\mathbb{R}^{m-k}$ non-including a whole straight line then the existence of $k$-wave factorization for the symbol $\tilde{A}(\cdot, \xi)$ with respect to the cone $C^{m-k}$ means the following. The symbol $A(\cdot, \xi)$ admits the representation

$$
A\left(\cdot, \xi^{\prime \prime}, \xi^{\prime}\right)=A_{\neq}\left(\cdot, \xi^{\prime \prime}, \xi^{\prime}\right) \cdot A_{=}\left(\cdot, \xi^{\prime \prime}, \xi^{\prime}\right),
$$

where $\xi^{\prime \prime}=\left(\xi_{1}, \cdot, \xi_{k}\right), \xi^{\prime}=\left(\xi_{k+1}, \cdot, \xi_{m}\right)$, factors $A_{\neq}\left(\cdot, \xi^{\prime \prime}, \xi^{\prime}\right), A_{=}\left(\cdot, \xi^{\prime \prime}, \xi^{\prime}\right)$ admit holomorphic continuation with re-

spect to the $\xi^{\prime}$ (for fixed $\xi^{\prime \prime}$ ) into radial tube domains $T\left(C^{m-k}\right), T\left(-C^{*-k}\right)$ respectively with some estimates in which the index ${ }_{k}$ of $k$-wave factorization takes part (one can find the rigorous definition in [13]). The value of the ${ }_{k}$ is essential for a solvability of corresponding conjugation problem.

Unfortunately, existence of $k$-wave factorization is one of main problems, that's why this is the third postulate.

\section{GENERAL CONCEPT: OPERATOR APPROACH}

As we have seen there are distinct types of "local operators" (2),(3),(4) for the operator (1). We will denote all such operators by unified formula

$$
u(x) \longmapsto \int_{D_{x_{0}}} A\left(x_{0}, x-y\right) u(y) d y, \quad x \in D_{x_{0}},
$$

meaning $D_{x_{0}}$ as one of above domains.

It is convenient to consider the operator $A$ on a manifold with such a family of local representatives in SobolevSlobodetskii spaces $H^{s}(D)$ for which its "local" variant will be the space $H^{s}\left(D_{x_{0}}\right)$. 
Definition 2 Symbol of the operator (1) is called the operator function $A(x): D \rightarrow\left\{A_{x}\right\}_{x \in D}$ which is a family of local representatives of the operator (1). The operator (1) is called an elliptic operator if its symbol consists of invertible operators.

In other words, symbol of the operator (1) is operator family (5) when $x_{0}$ varies in $\bar{D}$.

\section{Canonical Domains and Model Operators}

Definition 3 Canonical domains in Euclidean space $\mathbb{R}^{m}$ is such a domain which is diffeomorphic to one of the following cones: $\mathbb{R}^{m}, \mathbb{R}_{+}^{m}, \mathbb{R}^{k} \times C^{m-k}, k=0,1, \cdots, m-2$, where $C^{m-k}$ is a convex cone in $\mathbb{R}^{m-k}$ non-including a whole straight line.

Remark 1 If we put by definition $\mathbb{R}^{m} \times C^{0} \equiv \mathbb{R}^{m}, \mathbb{R}^{m-1} \times C^{1} \equiv \mathbb{R}_{+}^{m}, \mathbb{R}^{0} \times C^{m} \equiv C^{m}$ then an arbitrary canonical domain will be a cone of the type $\mathbb{R}^{k} \times C^{m-k}, k=0,1, \cdots, m-2, m-1, m$.

Definition 4 A model operator is called an operator of type (5) in some canonical domain with the symbol $\tilde{A}(\cdot, \xi)$ non-depending on a spatial variable.

\section{Manifolds with a Singular Boundary}

To define a domain (manifold) $D$ with a singular boundary we will introduce different types of neighborhoods. For a smooth compact manifold all neighborhoods $\left\{U_{j}\right\}$ have the same type, these are diffeomorhic to $\mathbb{R}^{m}$. But if a manifold has a smooth boundary even then there are two types of neighborhoods related to a place of a neighborhood, namely inner neighborhoods and boundary ones. For inner neighborhood $U$ such that $U \subset \stackrel{D}{ }$ we have the diffeomorphism $\omega: U \rightarrow \mathbb{R}^{m}$. For a boundary neighborhood such that $U \cap \partial D \neq \emptyset$ we have another diffeomorphism $\omega_{1}: U \rightarrow \mathbb{R}_{+}^{m}$. May be this boundary $\partial D$ has some singularities like conical points and wedges. A conical point at the boundary is such a point for which its neighborhood is diffeomorphic to the cone $C^{m}$, a wedge point of codimension $k, 1 \leq k \leq$ $m-2$, is such a point for which its neighborhood is diffeomorphic to the cone $\mathbb{R}^{k} \times C^{m-k}$. So if the manifold $D$ has such singularities we suppose that we can extract certain k-dimensional sub-manifolds, namely $(m-1)$-dimensional boundary $\partial D$, and $k$-dimensional wedges $D_{k}, k=0, \cdots, m-2 ; D_{0}$ is a collection of conical points.

\section{Operators on Manifolds}

If $D$ is a compact domain (manifold) then there is a partition of unity. It means the following. For every finite open covering $\left\{U_{j}\right\}_{j=1}^{k}$ of the manifold $D$ there exists a system of functions $\left\{\varphi_{j}(x)\right\}_{j=1}^{k}, \varphi_{j}(x) \in C^{\infty}(D)$, such that

- $0 \leq \varphi_{j}(x) \leq 1$,

- $\operatorname{supp} \varphi_{j} \subset U_{j}$,

- $\sum_{j=1}^{k} \varphi_{j}(x)=1$.

So we have

$$
f(x)=\sum_{j=1}^{k} \varphi_{j}(x) f(x)
$$

for arbitrary function $f$ defined on $D$.

Since an every set $U_{j}$ is diffeomorphic to an open set $D_{k}^{j}=\mathbb{R}^{k} \times C^{m-k}$ for some $k$ we have corresponding diffeomorphisms $\omega_{j}: U_{j} \rightarrow D_{k}^{j}$. Further for a function $f$ defined on $D$ we compose mappings $f_{j}=f \cdot \varphi_{j}$ and as far as supp $f_{j} \subset U_{j}$ we put $\hat{f}_{j}=f_{j} \circ \omega_{j}^{-1}$ so that $\hat{f}_{j}: D_{k}^{j} \rightarrow \mathbb{R}$ is a function defined in a domain of $m$-dimensional space $\mathbb{R}^{m}$.

On the manifold $D$ we fix a finite open covering and a partitions of unity corresponding to this covering $\left\{U_{j}, f_{j}\right\}_{j=1}^{n}$ and choose smooth functions $\left\{g_{j}\right\}_{j=1}^{n}$ so that supp $g_{j} \subset V_{j}, \overline{U_{j}} \subset V_{j}$, and $g_{j}(x) \equiv 1$ for $x \in \operatorname{supp} f_{j}$, supp $f_{j} \cap\left(1-g_{j}\right)=$ $\emptyset$. 
Definition 5 The operator $A$ on the manifold $D$ can be represented in the form

$$
A=\sum_{j=1}^{n} f_{j} \cdot \hat{A}_{j} \cdot g_{j}+T
$$

where $T: H^{s}(D) \rightarrow H^{s-\alpha}(D)$ is a compact operator, $\hat{A}_{j}$ is one of model operators $(5)$ in which the change of variables $\omega_{j}$ was done.

\section{Fredholm Properties}

Invertibility of local operators First conclusion from the local principle is the following.

Theorem 1 The operator A has Fredholm property iff it is an elliptic operator.

Let us denote ${ }_{k}(x)$ the indices of $k$-wave factorization of the symbol $A(x, \xi)$ with respect to the cone $C \stackrel{m-k}{ }$ at points $x \in M_{k}, k=0,1, \cdots, m-2$ and assume that the functions ${ }_{k}(x), k=0,1, \cdots, m-1$, can be continued into $M_{k}$ because it is possible $M_{k} \cap M_{k-1} \neq \emptyset$.

Proposition 1 Let the classical symbol $A(x, \xi)$ admits $k$-wave factorization with respect to the cone $C^{m-k}$ with indices ${ }_{k}(x), k=0,1, \cdots, m-2$ satisfying the conditions

$$
|k(x)-s|<1 / 2, \quad \forall x \in M_{k}, \quad k=0,1, \cdots, m-1 .
$$

Then the operator $A: H^{s}(M) \rightarrow H^{s-\alpha}(M)$ has Fredholm property.

Remark 2 Let us note that $(m-1)$-wave factorization is usual factorization in Eskin's sense and it always exists [19].

On boundary conditions Let us suppose that one of conditions (6) does not hold. It means that there is at list one point $x_{o} \in \partial D$. If $x_{0}$ is a smoothness point at the boundary $\partial D$ then you can look [19]. We suppose here that $x_{0}$ is a conical point so that $D_{x_{0}}=C$ and for this this convex cone $C$ its boundary is given by the equation $x_{m}=\varphi\left(x^{\prime}\right)$. Moreover we suppose that $0\left(x_{0}\right)-s=n+\varepsilon,|\varepsilon|<1 / 2$. Then the local representative $A_{x_{0}}$ is not invertible, but it is possible to describe its kernel [16, 17].

Let $C$ be a convex cone in the space $\mathbb{R}^{m}$, and this cone does not include any whole straight line, it is important because we use the theory of analytic functions of several complex variables [21, 22, 23]. Moreover we suppose that a surface of this cone is given by the equation $x_{m}=\varphi\left(x^{\prime}\right), x^{\prime}=\left(x_{1}, \cdots, x_{m-1}\right)$, where $\varphi: \mathbb{R}^{m-1} \rightarrow \mathbb{R}$ is a smooth function in $\mathbb{R}^{m-1} \backslash\{0\}$, and $\varphi(0)=0$.

Let us introduce the following change of variables

$$
\left\{\begin{aligned}
t_{1} & =x_{1} \\
t_{2} & =x_{2} \\
\cdots & \\
t_{m-1} & =x_{m-1} \\
t_{m} & =x_{m}-\varphi\left(x^{\prime}\right)
\end{aligned}\right.
$$

and we denote this operator by $T_{\varphi}: \mathbb{R}^{m} \rightarrow \mathbb{R}^{m}$ so that points from lower half-space will be fixed.

Theorem 2 A kernel of the operator $A_{x_{0}}$ in the space $H^{s}(C)$ consists of functions of the following type

$$
A_{\neq}^{-1}(\xi) V_{-\varphi} F\left(\sum_{k=0}^{n-1} c_{k}\left(x^{\prime}\right) \delta^{(k)}\left(x_{m}\right)\right),
$$

where $A_{\neq}(\xi)$ is factor of 0 -wave factorization for the symbol $A\left(x_{0}, \xi\right), \delta$ is the Dirac mass-function, and $V_{-\varphi}$ is a special integral operator defined by changes of variables in m-dimensional space and the Fourier transform, $c_{k}\left(x^{\prime}\right)$ are arbitrary functions from the space where $H^{s_{k}}\left(\mathbf{R}^{m-1}\right), s_{k}=s-{ }_{0}\left(x_{0}\right)+k+1 / 2, k=0,1,2, \ldots, n-1$.

We have the a priori estimate

$$
\|u\|_{s} \leq \mathrm{const} \sum_{k=0}^{n-1}\left[c_{k}\right]_{s_{k}}
$$

where $[\cdot]_{s_{k}}$ denotes the $H^{s_{k}}\left(\mathbb{R}^{m-1}\right)$-norm. 
Therefore, to obtain invertibility for such operator $A_{x_{0}}$ and to annihilate the kernel we need to add some additional conditions. As a rule such conditions are chosen as boundary conditions.

Remark 3 It is very unpleasant but it may be happened that we need distinct number of conditions for different placements of the point $x_{0}$. But I think such a problem is now very far from nearest ones.

\section{DESCRIPTION OF OPERATOR $V_{\varphi}$ AND ITS PROPERTIES}

To obtain explicit expression for $V_{\varphi}$ we need to choice a concrete cone. We consider here $C \equiv C_{+}^{a}=\left\{x \in \mathbb{R}^{m}: x=\right.$ $\left.\left(x^{\prime}, x_{m}\right), x_{m}>a\left|x^{\prime}\right|, a>0\right\}$, the notation $T_{\varphi} \equiv T_{a}, V_{\varphi} \equiv V_{a}$ and use the transmutation formula

$$
F T_{a}^{-1}=V_{-a} F .
$$

Let us fix $u \in S\left(\mathbb{R}^{m}\right)$ and evaluate

$$
\begin{aligned}
& \left(F T_{a}^{-1} u\right)(\xi)=\int_{\mathbb{R}^{m}} e^{i y \cdot \xi}\left(T_{a}^{-1} u\right)(y) d y=\int_{\mathbb{R}^{m}} e^{i y \cdot \xi^{\prime}} u\left(y^{\prime}, y_{m}+a\left|y^{\prime}\right|\right) d y \\
& =\int_{\mathbb{R}^{m}} e^{i x \cdot \xi} e^{-i \xi_{m} a\left|x^{\prime}\right|} u\left(x^{\prime}, x_{m}\right) d x^{\prime} d x_{m}=\int_{\mathbb{R}^{m}} e^{i x^{\prime} \cdot \xi^{\prime}} e^{-i \xi_{m} a\left|x^{\prime}\right|} \hat{u}\left(x^{\prime}, \xi_{m}\right) d x^{\prime},
\end{aligned}
$$

where $\hat{u}\left(x^{\prime}, \xi_{m}\right)$ denotes the Fourier transform of the function $u\left(x^{\prime}, x_{m}\right)$ with respect to the variable $x_{m}$. According to properties of the Fourier transform we denote

$$
F_{x^{\prime} \rightarrow \xi^{\prime}}\left(e^{-i \xi_{m} a\left|x^{\prime}\right|}\right) \equiv K_{a}\left(\xi^{\prime}, \xi_{m}\right),
$$

and obtain an integral representation for the operator $V_{-a}$ :

$$
\left(F T_{a}^{-1} u\right)(\xi)=\int_{\mathbb{R}^{m}} K_{a}\left(\xi^{\prime}-\eta^{\prime}, \xi_{m}\right) \tilde{u}\left(\eta^{\prime}, \xi_{m}\right) d \eta^{\prime}
$$

where $K_{a}\left(\xi^{\prime}, \xi_{m}\right)$ is the Fourier image of corresponding distribution.

Since the function $e^{-i \xi_{m} a\left|x^{\prime}\right|}$ is not integrable in $\mathbb{R}^{m-1}$ we need some regularization method, and we mean the latter formula as follows

$$
\lim _{\tau \rightarrow 0} F_{x^{\prime} \rightarrow \xi^{\prime}}\left(e^{-i z_{m} a\left|x^{\prime}\right|}\right), \quad z_{m}=\xi_{m}-i \tau, \quad \tau>0
$$

so that

$$
\left(F T_{a}^{-1} u\right)(\xi)=\lim _{\tau \rightarrow 0+} \int_{\mathbb{R}^{m}} K_{a}\left(\xi^{\prime}-\eta^{\prime}, z_{m}\right) \tilde{u}\left(\eta^{\prime}, \xi_{m}\right) d \eta^{\prime}
$$

According to above

$$
F_{x^{\prime} \rightarrow \xi^{\prime}}\left(e^{-i z_{m} a\left|x^{\prime}\right|}\right)=\int_{\mathbb{R}^{m-1}} e^{i x^{\prime} \cdot \xi^{\prime}} e^{-i z_{m} a\left|x^{\prime}\right|} d x^{\prime}
$$

and we cam use certain technical methods developed in [24] to find the following representation.

Proposition 2 The function $K_{a}$ has the following form

$$
K_{a}\left(\xi^{\prime}, z_{m}\right)=\frac{i a z_{m} 2^{m-1} \pi^{\frac{m-2}{2}} \Gamma(m / 2)}{\left(\xi_{1}^{2}+\xi_{2}^{2}+\cdots \xi_{m-1}^{2}-a^{2} z_{m}^{2}\right)^{m / 2}},
$$

where $z_{m}=\xi_{m}-i \tau, \tau>0$. 
So, we have

$$
\left(V_{-a} \tilde{u}\right)(\xi)=\lim _{\tau \rightarrow 0+} \frac{1}{\pi^{m / 2}} \int_{\mathbb{R}^{m-1}} \frac{i a z_{m} \Gamma(m / 2) \tilde{u}\left(\eta^{\prime}, \xi_{m}\right) d \eta^{\prime}}{\left(\left|\xi^{\prime}-\eta^{\prime}\right|^{2}-a^{2} z_{m}^{2}\right)^{m / 2}}
$$

Corollary 1 If $a \rightarrow 0$ then

$$
\left(V_{0} \tilde{u}\right)(\xi)=\lim _{a \rightarrow 0} \lim _{\tau \rightarrow 0+} \frac{1}{\pi^{m / 2}} \int_{\mathbb{R}^{m-1}} \frac{i a z_{m} \Gamma(m / 2) \tilde{u}\left(\eta^{\prime}, \xi_{m}\right) d \eta^{\prime}}{\left(\left|\xi^{\prime}-\eta^{\prime}\right|^{2}-a^{2} z_{m}^{2}\right)^{m / 2}}=\tilde{u}\left(\xi^{\prime}, \xi_{m}\right) .
$$

Corollary 2 If $\xi_{m} \rightarrow 0$ then

$$
\lim _{\xi_{m} \rightarrow 0}\left(V_{-a} \tilde{u}\right)(\xi)=\lim _{\xi_{m} \rightarrow 0} \lim _{\tau \rightarrow 0+} \frac{1}{\pi^{m / 2}} \int_{\mathbb{R}^{m-1}} \frac{i a z_{m} \Gamma(m / 2) \tilde{u}\left(\eta^{\prime}, \xi_{m}\right) d \eta^{\prime}}{\left(\left|\xi^{\prime}-\eta^{\prime}\right|^{2}-a^{2} z_{m}^{2}\right)^{m / 2}}=\tilde{u}\left(\xi^{\prime}, 0\right)
$$

Theorem 3 The kernel of the equation (5) consists of functions of the following type

$$
\tilde{u}(\xi)=A_{\neq}^{-1}(\xi)\left(\sum_{k=1}^{n} \tilde{C}_{k}\left(\xi, \xi_{m}\right) \xi_{m}^{k-1}\right)
$$

where

$$
\tilde{C}_{k}\left(\xi, \xi_{m}\right)=\left(V_{-a} \tilde{c}_{k}\right)\left(\xi^{\prime}, \xi_{m}\right)
$$

Thus, for unique identification of the functions $\tilde{C}_{k}\left(\xi^{\prime}, \xi_{m}\right)$ we need some additional conditions. We consider some variants below.

\section{Case $m=2$}

First, we need to note that 2-dimensional case was studied without operator $V_{\varphi}$, but to verify this way we have constructed the operator $V_{\varphi}$ for this case also. Second, for 2-dimensional case we have only one type of a cone (excluding rotations) $C_{+}^{a}=\left\{x \in \mathbb{R}^{2}: x=\left(x_{1}, x_{2}\right), x_{2}>a\left|x_{1}\right|, a>0\right\}$. Thus, we have the following boundary function $\varphi\left(x_{1}\right)=a\left|x_{1}\right|$. For this function we have evaluated the operator $V_{\varphi}$ [16] using analytic properties of the Fourier transform, and it looks as follows

$$
\begin{gathered}
\left(V_{a} \tilde{u}\right)(\xi)=\frac{\tilde{u}\left(\xi_{1}+a \xi_{2}, \xi_{2}\right)+\tilde{u}\left(\xi_{1}-a \xi_{2}, \xi_{2}\right)}{2} \\
+v \cdot p \cdot \frac{i}{2 \pi} \int_{-\infty}^{+\infty} \frac{\tilde{u}\left(\eta, \xi_{2}\right) d \eta}{\xi_{1}+a \xi_{2}-\eta}-v \cdot p \cdot \frac{i}{2 \pi} \int_{-\infty}^{+\infty} \frac{\tilde{u}\left(\eta, \xi_{2}\right) d \eta}{\xi_{1}-a \xi_{2}-\eta} .
\end{gathered}
$$

\section{Case $m=3$}

We have a lot of cones in this case, and we consider some of them. 
4-sided angle. The boundary function for this case is the following $\varphi(x)=a_{1}\left|x_{1}\right|+a_{2}\left|x_{2}\right|$, i.e. the conical surface is described by the equation $x_{2}=a_{1}\left|x_{1}\right|+a_{2}\left|x_{2}\right|$. The cone looks as follows $C_{a_{1} a_{2}}=\left\{x \in \mathbb{R}^{3}: x_{2}>a_{1}\left|x_{1}\right|+a_{2}\left|x_{2}\right|\right\}$. Let us introduce two operators defined on functions of three variables

$$
\begin{aligned}
& \left(S_{1} u\right)\left(\xi_{1}, \xi_{2}, \xi_{3}\right)=v \cdot p \frac{i}{2 \pi} \int_{-\infty}^{+\infty} \frac{u\left(\tau, \xi_{2}, \xi_{3}\right) d \tau}{\xi_{1}-\tau}, \\
& \left(S_{2} u\right)\left(\xi_{1}, \xi_{2}, \xi_{3}\right)=v \cdot p \frac{i}{2 \pi} \int_{-\infty}^{+\infty} \frac{u\left(\xi_{1}, \eta, \xi_{3}\right) d \eta}{\xi_{2}-\eta} .
\end{aligned}
$$

If we will denote corresponding operator by $T_{a_{1} a_{2}}$ (change of variables) and its transmutation operator by $V_{a_{1} a_{2}}$ then we obtain

$$
\begin{aligned}
\left(V_{a_{1} a_{2}} \tilde{u}\right)\left(\xi_{1}, \xi_{2}, \xi_{3}\right)=\frac{\tilde{u}\left(\xi_{1}-a_{1} \xi_{3}, \xi_{2}-a_{2} \xi_{3}, \xi_{3}\right)+\tilde{u}\left(\xi_{1}+a_{1} \xi_{3}, \xi_{2}-a_{2} \xi_{3}, \xi_{3}\right)}{4} & \frac{1}{2}\left(S_{1} \tilde{u}\right)\left(\xi_{1}+a_{1} \xi_{3}, \xi_{2}-a_{2} \xi_{3}, \xi_{3}\right)-\frac{1}{2}\left(S_{1} \tilde{u}\right)\left(\xi_{1}-a_{1} \xi_{3}, \xi_{2}-a_{2} \xi_{3}, \xi_{3}\right) \\
& +\frac{\tilde{u}\left(\xi_{1}-a_{1} \xi_{3}, \xi_{2}+a_{2} \xi_{3}, \xi_{3}\right)+\tilde{u}\left(\xi_{1}+a_{1} \xi_{3}, \xi_{2}+a_{2} \xi_{3}, \xi_{3}\right)}{4} \\
+ & \frac{1}{2}\left(S_{1} \tilde{u}\right)\left(\xi_{1}+a_{1} \xi_{3}, \xi_{2}+a_{2} \xi_{3}, \xi_{3}\right)-\frac{1}{2}\left(S_{1} \tilde{u}\right)\left(\xi_{1}-a_{1} \xi_{3}, \xi_{2}+a_{2} \xi_{3}, \xi_{3}\right) \\
& +\frac{\left(S_{2} \tilde{u}\right)\left(\xi_{1}-a_{1} \xi_{3}, \xi_{2}+a_{2} \xi_{3}, \xi_{3}\right)+\left(S_{2} \tilde{u}\right)\left(\xi_{1}+a_{1} \xi_{3}, \xi_{2}+a_{2} \xi_{3}, \xi_{3}\right)}{2} \\
+ & \left(S_{1} S_{2} \tilde{u}\right)\left(\xi_{1}+a_{1} \xi_{3}, \xi_{2}+a_{2} \xi_{3}, \xi_{3}\right)-\left(S_{1} S_{2} \tilde{u}\right)\left(\xi_{1}-a_{1} \xi_{3}, \xi_{2}+a_{2} \xi_{3}, \xi_{3}\right) \\
& -\frac{\left(S_{2} \tilde{u}\right)\left(\xi_{1}-a_{1} \xi_{3}, \xi_{2}-a_{2} \xi_{3}, \xi_{3}\right)-\left(S_{2} \tilde{u}\right)\left(\xi_{1}+a_{1} \xi_{3}, \xi_{2}-a_{2} \xi_{3}, \xi_{3}\right)}{2} \\
- & \left(S_{1} S_{2} \tilde{u}\right)\left(\xi_{1}+a_{1} \xi_{3}, \xi_{2}-a_{2} \xi_{3}, \xi_{3}\right)+\left(S_{1} S_{2} \tilde{u}\right)\left(\xi_{1}-a_{1} \xi_{3}, \xi_{2}-a_{2} \xi_{3}, \xi_{3}\right) .
\end{aligned}
$$

This case was studied in [17] (see also [25]) and here we give the final form only

Circle cone. According to the formula (7) we have for $C_{+}^{a}$

$$
\left(V_{-a} \tilde{u}\right)\left(\xi_{1}, \xi_{2}, \xi_{3}\right)=\lim _{\tau \rightarrow 0+} \frac{1}{\pi^{3 / 2}} \int_{\mathbb{R}^{2}} \frac{i a z_{3} \Gamma(3 / 2) \tilde{u}\left(\eta_{1}, \eta_{2}, \xi_{3}\right) d \eta_{1} d \eta_{2}}{\left(\left(\xi_{1}-\eta_{1}\right)^{2}+\left(\xi_{2}-\eta_{2}\right)^{2}-a^{2} z_{3}^{2}\right)^{3 / 2}}
$$

\section{Multidimensional and Complicated Cases}

There are a lot of cones in multidimensional Euclidean space which can be constructed from those that we have considered. We will enumerate some of them.

- $\mathbb{R}^{k} \times C_{+}^{a} \subset \mathbb{R}^{k+m}$;

- $C_{a_{1} a_{2}} \times C_{+}^{a} \subset \mathbb{R}^{m+3}$;

- $(0,+\infty) \times C_{+}^{a} \subset \mathbb{R}^{m+1}$;

- $(0,+\infty) \times C_{+}^{a} \times C_{a_{1} a_{2}} \subset \mathbb{R}^{m+4}$;

- $\mathbb{R}^{k} \times(0,+\infty) \times C_{+}^{a} \times C_{a_{1} a_{2}} \subset \mathbb{R}^{k+m+4}$.

For every of these cases we will obtain special type of the integral operator $V_{\varphi}$, and every particular case will generate its own formula for a general solution. We will consider such possibilities in forthcoming publications. 


\section{INTEGRAL CONDITION AND SOLVABILITY}

Taking into account Theorem 2 we consider the equation with a model operator

$$
(A u)(x)=0, \quad x \in C_{+}^{a},
$$

in the cone $C_{+}^{a}$ with symbol $A(\xi)$ for $n=1$. Then we have the following formula for a general solution in view of (7)

$$
\tilde{u}(\xi)=A_{\neq}^{-1}(\xi) V_{-a} \tilde{c}_{0}\left(\xi^{\prime}\right)
$$

with an arbitrary function $c_{0} \in H^{s-+1 / 2}\left(\mathbb{R}^{m-1}\right)$. Let us suppose that the following condition

$$
\int_{-\infty}^{+\infty} u\left(x^{\prime}, x_{m}\right) d x_{m}=g\left(x^{\prime}\right)
$$

where $g\left(x^{\prime}\right)$ is given function from $H^{s+1 / 2}\left(\mathbb{R}^{m-1}\right)$. According to the Fourier transform properties we have

$$
\tilde{u}\left(\xi^{\prime}, 0\right)=\tilde{g}\left(\xi^{\prime}\right),
$$

and taking into account Corollary 2 we can find $\tilde{c}_{0}\left(\xi^{\prime}\right)$ immediately from the equality

$$
\tilde{u}\left(\xi^{\prime}, 0\right)=A_{\neq}^{-1}\left(\xi^{\prime}, 0\right) \tilde{c}_{0}\left(\xi^{\prime}\right),
$$

more exactly

$$
\tilde{c}_{0}\left(\xi^{\prime}\right)=A_{\neq}\left(\xi^{\prime}, 0\right) \tilde{g}\left(\xi^{\prime}\right) .
$$

Therefore, we can formulate the following result.

Theorem 4 The boundary value problem (8), (9) has unique solution in the space $H^{s}\left(C_{+}^{a}\right)$ for arbitrary given function $g\left(x^{\prime}\right) \in H^{s+1 / 2}\left(\mathbb{R}^{m-1}\right)$ of the following type

$$
\tilde{u}\left(\xi^{\prime}, \xi_{m}\right)=\frac{A_{\neq}^{-1}\left(\xi^{\prime}, \xi_{m}\right)}{\pi^{m / 2}} \lim _{\tau \rightarrow 0+} \int_{\mathbb{R}^{m-1}} \frac{i a z_{m} \Gamma(m / 2) A_{\neq}\left(\eta^{\prime}, 0\right) \tilde{g}\left(\eta^{\prime}\right) d \eta^{\prime}}{\left(\left|\xi^{\prime}-\eta^{\prime}\right|^{2}-a^{2} z_{m}^{2}\right)^{m / 2}}, \quad z_{m}=\xi_{m}-i \tau, \quad \tau>0 .
$$

A priori estimate

$$
\|u\|_{s} \leq \text { const }[g]_{s+1 / 2}
$$

holds.

\section{CONCLUSION}

Here we have tried to show how much possibilities gives the developed approach, and how we can find some integral representations for certain boundary value problems. General concepts of this approach was described in author's papers $[26,27]$ but as you see there are a lot of difficulties to realize these ideas. But we hope that this point of view can be useful and can give a new impulse to develop the theory.

\section{REFERENCES}

1. V. Kozlov, V. Maz’ya, and J. Rossmann, Spectral Problems Associated with Corner Singularities of Solutions to Elliptic Equations (AMS, Providence, RI, 2001).

2. S. Nazarov and B. A. Plamenevsky, Elliptic Problems in Domains with Piecewise Smooth Noundaries (Walter de Gruyter, Berlin - New York, 1994). 
3. V. E. Nazaikinskii, A. Y. Savin, B.-W. Schulze, and B. Y. Sternin, Elliptic Theory on Singular Manifolds (Chapman \& Hall/CRC, Boca Raton, 2006).

4. J. Egorov and B.-W. Schulze, Pseudo-Differential Operators, Singularities, Applications (Birkhäuser-Verlag, Basel, 1997).

5. B.-W. Schulze, Boundary Value Problems and Singular Pseudo-Differential Operators (J. Wiley, Chichester, 1998).

6. B.-W. Schulze, B. Sternin, and V. Shatalov, Differential Equations on Singular Manifolds; Semiclassical Theory and Operator Algebras (Wiley-VCH, Berlin, 1998).

7. A. Dynin, Proc. Nat. Acad. Sci. USA 75, 4668-4670 (1978).

8. A. Dynin, Integr Equat. Oper. Theory 9, 537-556 (1986).

9. A. Dynin, Integr Equat. Oper. Theory 10, 554-576 (1987).

10. B. Sternin, Trudy Moskov. Mat. Obsh. (in Russian) 15, 346-382 (1966).

11. V. B. Vasilyev, "Asymptotical analysis of singularities for pseudo differential equations in canonical non-smooth domains," in Integral Methods in Science and Engineering. Computational and Analytic Aspects, edited by C. Constanda and P. J. Harris (Birkhauser, Boston, 2011) pp. 379390.

12. V. Vasilyev, Bound. Value Probl. 116, 1-14 (2017).

13. V. Vasil'ev, Wave Factorization of Elliptic Symbols: Theory and Applications. Introduction to the Theory of Boundary Value Problems in Non-Smooth Domains (Kluwer Academic Publishers, Dordrecht-Boston-London, 2000).

14. V. Vasilyev, Math. Bohem. 39, 333-340 (2014).

15. V. Vasilyev, Adv. Dyn. Syst. Appl. 9, 227-237 (2014).

16. V. Vasilyev, Opusc. Math. 39, 109-124 (2019).

17. V. Vasilyev, Math. Meth. Appl. Sci. 41, 9252-9263 (2018).

18. V. B. Vasilyev, "Pseudo-differential operators on manifolds with a singular boundary," in Modern Problems in Applied Analysis, edited by P. Drygas and S. Rogosin (Birkhauser, Cham, 2018) pp. 169-179.

19. G. Eskin, Boundary Value Problems for Elliptic Pseudodifferential Equations (AMS, Providence, RI, 1981).

20. I. Simonenko, Local Method in the Theory of Translation Invariant Operators and Their Envelopes (CVVR (in Russian), Rostov on Don, 2007).

21. V. Vladimirov, Methods of the Theory of Functions of Many Complex Variables (Dover Publications, Mineola, NY, 2007).

22. V. Vladimirov, Generalized Functions in Mathematical Physics (Mir Publishers, Moscow, 1979).

23. S. Bochner and W. Martin, Several Complex Variables (Princeton Univ. Press, Princeton, NY, 1948).

24. E. M. Stein and G. Weiss, Introduction to Fourier Analysis on Euclidean Spaces (Princeton Univ. Pres, Princeton, 1971).

25. V. Vasilyev, Lobachevskii J. Math, 41, 917-925 (2020).

26. V. Vasilyev, Demonstr. Math. 52, 361-369 (2019).

27. V. Vasilyev, Symmetry 64, 1-12 (2020). 